\title{
Measures of functional brain connectivity can be used to predict outcome after glioma surgery
}

Magnetoencephalography (MEG) in patients with glioma could represent a tool to predict long-term surgical outcome, according to a new study by researchers at the University of California, San Francisco, CA, USA. "We hypothesized that tumours in regions of the brain with low resting-state functional connectivity could be removed with very little impact on long-term functional outcomes," says Srikantan Nagarajan, who led the study.

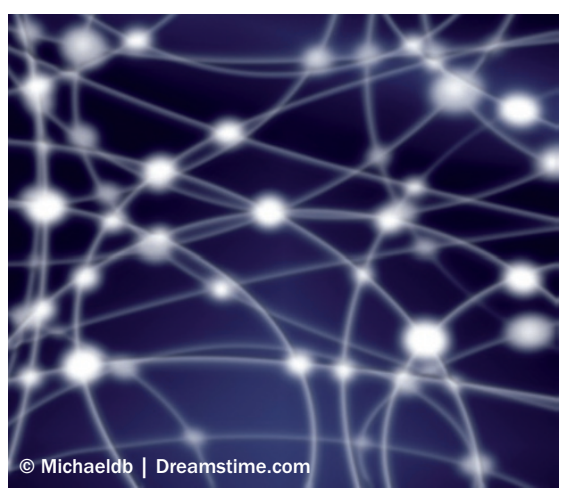

Resection of brain tumours located in or near cortical areas involved in language, motor or sensory functions carries the risk of lasting neurological damage. As the tumour mass can alter cerebral topography, classic anatomical maps are unsuitable for surgical planning.

Nagarajan and colleagues used wholebrain MEG to compare presurgical resting-state connectivity of lesional versus contralesional areas to various brain regions. The study involved 79 consecutive patients with focal brain gliomas.

In patients with tumours with low connectivity to other brain regions, $28.6 \%$ showed neurological morbidity 1 week after surgery, dropping to $0 \%$ after 6 months. By contrast, patients with tumours showing high-level functional connectivity had a $60 \%$ chance of neurological deficits 1 week postsurgery, and a $25 \%$ chance of such morbidity at 6-month follow-up. In patients with equal lesional and contralesional connectivity, risk of surgery-associated neurological impairment was between that of the lowconnectivity and high-connectivity groups.

MEG-based connectivity analysis using MEG could be useful for planning surgical strategies for glioma and for informing patients of postoperative risks. "Our findings highlight the importance of using novel functional brain imaging modalities to gain insights into brain networks that are affected by brain lesions or by targeted treatments of the brain," says Nagarajan. His group is looking to extend the results to active-state mapping of functional connectivity in tumours, as well as examining radiation therapy.

\section{Katie Kingwell}

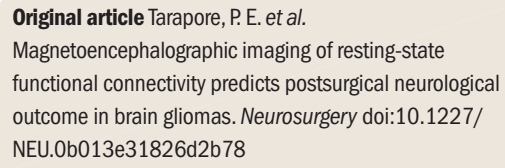

